\title{
A Link between Mitochondrial DNA Haplogroup and Ischemia
}

\author{
Seyed E. Hasnain \\ Institute of Life Sciences, Hyderabad University Campus, Hyderabad, and Jawaharlal Nehru Centre \\ for Advanced Scientific Research, Bangalore, India
}

Genomic medicine or gene-based medicine, terms coined to describe a paradigm shift in human health, as a consequence of the Human Genome Project [1], is under retrospection to assess the true impact of this genome revolution [2]. The tangible gains of genomic medicine have resulted in a better understanding of the molecular pathways leading to disease manifestations and the importance of genes and specific mutations therein in terms of drug response and disease progression and in assessing the risk of contracting disease. Individual genetic ancestry has been found to improve predictions of pulmonary function in self-identified African Americans [3] pointing to the significance of 'race correction' in pulmonary function testing [4]. These genetic markers are the prime gain of the genomic revolution, even though questions of accuracy of such predictions remain. While single-nucleotide polymorphisms (SNPs) in the nuclear genome continue to be the focus of disease association, enough reports highlight the importance of nonnuclear SNPs, the epigenetic phenomenon and the environment as risk factors.

The mitochondrial respiratory chain, the most fundamental and key process for cellular energy, is vital for normal functioning of an organism. Any disruption caused by mutations in the genes encoding critical enzymes in the respiratory pathway, present in the mitochondrial or nuclear genome, has a major impact on a diverse group of

\section{KARGER}

Fax +4161306 1234

E-Mail karger@karger.ch

www.karger.com
(C) 2011 S. Karger AG, Basel

1011-7571/11/0203-0201\$38.00/0

Accessible online at:

www.karger.com/mpp diseases collectively known as mitochondrial disease and including multisystem disorders. Mitochondrial DNA (mtDNA) mutations also manifest in disturbing the reactive oxygen species balance in the cell, leading to diverse pathological conditions.

The maternally inherited mtDNA does not undergo biparental recombination but accumulating polymorphisms have been forming branches of the phylogenetic tree throughout human history. mtDNA polymorphism is far more robust in terms of its discerning power than nuclear SNPs. These so-called mtDNA haplogroups represent major subdivisions of phylogeny, which diversified as a function of geographic migrations of humans, thousands of years ago, creating region-specific haplogroup variations.

Mitochondria, almost exclusively maternally inherited, have been the subject of investigation for diseases that have a sex bias. It is known that women have a greater chance of inheriting a stroke than men, consistent with the maternally transmitted risk allele [5]. An earlier report documented the role of pathogenic mtDNA mutations in a maternally inherited disease causing stroke-like symptoms, MELAS (mitochondrial encephalomyopathy with lactic acidosis and stroke-like episodes), wherein the cerebral vasculature showed biochemical and structural abnormalities [6]. Other reports found an association between mtDNA genetic variation and risk factors for isch- 
emic stroke and also hypertension, abnormal LDL levels, obesity and syndrome $\mathrm{X}$, that impact on mortality and longevity in the general population $[7,8]$.

In a recent study Chinnery et al. [9] showed evidence of an association between mtDNA haplogroups and incidence of stroke. In a case-control study with two independent disease cohorts $(n=950)$ and several independent control groups of all white individuals residing in the Oxford, UK, area, the occurrence of transient ischemic attack (TIA) and ischemic stroke was compared with control groups comprising of patients with acute coronary syndrome from the same $(n=340)$ or independent $(\mathrm{n}=2,939)$ population. For mtDNA haplogroup analyses, genotyping was carried out by matrix-associated laser desorption/ionization time of flight (MALDITOF) mass spectrometry followed by sequencing of random samples that generated $100 \%$ concordance. The frequency of subhaplogroup $\mathrm{K}$ was significantly lower in TIA/stroke patients than in patients with acute coronary syndromes ( $p=0.0098)$, healthy UK controls $(p=0.0082)$, and the combined control cohort of healthy and disease controls ( $\mathrm{p}=0.0066$ ). The less frequent presence, as compared to controls, of mtDNA subhaplogroup $\mathrm{K}$ in patients with TIA or stroke (odds ratio $0.54,95 \%$ CI $0.39-$ $0.75, \mathrm{p}<0.0001)$ remained significant even after adjusting for multiple haplogroup patients. Furthermore, the genetic association was highly significant for patients with TIA and stroke separately and appeared to be independent of known risk factors and for patients with acute coronary events. The subhaplogroup $\mathrm{K}$ was present in $8.7 \%$ of the control population, conferring a $4 \%(95 \%$ CI $2.2-5.7)$ reduction in population-attributed risk of TIA stroke.

An earlier study involving a Danish population [8] showed no evidence of association between haplogroup $\mathrm{K} 1$ and stroke or cardiovascular disease. The apparent contradiction between this and the present study is due to use of accurate brain imaging or autopsy and intense clinical follow-up involving a much larger population as opposed to a smaller cohort size and dependence purely on case note review.

The other rather surprising observation was the lack of any association between the maternal history of stroke and subhaplogroup K. Interestingly, previous meta-analyses of data on inheritance of ischemic stroke as a function of gender [5] revealed that despite the fact that mtDNA is passed on by the mother to the children, irrespective of gender, the sisters of women with stroke were at greater risk of stroke than their male siblings. It therefore becomes clear that mtDNA may not be exclusively responsible for the increased heritability of stroke in women, pointing to the role of sex-specific autosomal genetic factors, epigenetic mechanisms or even nongenetic mechanisms. While the precise mechanism behind the functional consequence of mtDNA haplogroup DNA variation for cerebrovascular disease remains unclear, this study certainly is a step forward to identify individuals at risk of ischemic stroke in the general population.

\section{References}

$\checkmark 1$ Varmus HE: Getting ready for gene-based medicine. N Engl J Med 2002;347:1526-1527.

$\checkmark 2$ Collins F: Has the gene revolution arrived? Nature 2010;464:674-675.

-3 Kumar R, Aldrich MC, Reiner AP: Genetic ancestry in lung-function predictions. N Engl J Med 2010;363:321-330.

-4 Scanlon PD, Shriver MD: 'Race correction' in pulmonary-function testing. $\mathrm{N}$ Engl J Med 2010;363:321-330.
5 Touze E, Rothwell PM: Sex differences in heritability of ischemic stroke; a systematic review and meta-analysis. Stroke 2008;39: $16-13$.

-6 Hirano M, Ricci E, Koenigsberger MR, et al: MELAS: an original case and clinical criteria for diagnosis. Neuromuscul Disord 1992;2: 125-135.

7 Tanaka M, Fuku N. Nishigaki Y: Women with mitochondrial haplogroup N9a are protected against metabolic syndrome. Diabetes 2007;56:518-521.
8 Benn M, Schwartz M, Nordestgaard BG, Tybjaerg-Hansen A: Mitochondrial haplogroups: ischemic cardiovascular disease, other diseases, mortality, and longevity in the general population. Circulation 2008; 117:2492-2501

-9 Chinnery PE, Elliot HR, Syed A, Rothwell PM: Mitochondrial DNA haplogroups and risk of transient ischaemic attack and ischaemic stroke: a genetic association study. Lancet Neurol 2010;9:498-503. 